\title{
Notas sobre a distribuição, tamanho de grupo e comportamento do golfinho Tursiops truncatus (Cetacea: Delphinidae) na Ilha de Santa Catarina, sul do Brasil
}

\author{
Leonardo Liberali Wedekin ${ }^{1,2,4}$, Fábio Gonçalves Daura-Jorge ${ }^{1,2}$, \\ Marcos Roberto Rossi-Santos ${ }^{2,3}$ \& Paulo César Simões-Lopes ${ }^{1}$ \\ ${ }^{1}$ Laboratório de Mamíferos Aquáticos - LAMAQ, Departamento de Ecologia e Zoologia, \\ Universidade Federal de Santa Catarina - UFSC, \\ Campus Universitário, s/n, CEP 88040-970, Florianópolis, SC, Brasil \\ ${ }^{2}$ Pós-graduação em Zoologia, Universidade Federal do Paraná - UFPR, Curitiba, PR, Brasil \\ ${ }^{3}$ Instituto Baleia Jubarte, \\ Rua Barão do Rio Branco, 26, CEP 45900-000, Caravelas, BA, Brasil \\ ${ }^{4}$ Autor para correspondência: Leonardo Wedekin, e-mail: lwedekin@hotmail.com
}

WEDEKIN, L.L., DAURA-JORGE, F.G., ROSSI-SANTOS, M.R. \& SIMÕES-LOPES, P.C. 2008. Notes on the distribution, group size and behavior of the bottlenose dolphin, Tursiops truncatus (Cetacea: Delphinidae) in the coast of the Island of Santa Catarina, southern Brazil. Biota Neotrop. 8(4): http://www.biotaneotropica. org.br/v8n4/en/abstract?short-communication+bn01708042008.

Abstract: The ecology of the bottlenose dolphin (Tursiops truncatus) is scarcely documented in Brazil. The objective of this article is to present information about the distribution, group size and behavior of T. truncatus collected oportunistically around the Island of Santa Catarina. Locality, date and time, group size and behavior were registered after each opportunistic sighting. Seventy-one groups were observed between 1989 and 2005, in all months of the year. The species was sighted along all the coast of the island, using a great variety of habitats including protected bays and exposed beaches. Group size varied from 1 to 200 individuals, with a mean group size of approximately 8 individuals (mode $=2$ ). Interactions with three species of sea birds were also documented. Data presented here suggest that the species is common around the Island of Santa Catarina, has a great plasticity in habitat use, and a varied behavior repertoire.

Keywords: bottlenose dolphin, Tursiops truncatus, behavior, group size, seabirds.

WEDEKIN, L.L., DAURA-JORGE, F.G., ROSSI-SANTOS, M.R. \& SIMÕES-LOPES, P.C. 2008. Notas sobre a distribuição, tamanho de grupo e comportamento do golfinho Tursiops truncatus (Cetacea: Delphinidae) na Ilha de Santa Catarina, sul do Brasil. Biota Neotrop. 8(4): http://www.biotaneotropica.org.br/v8n4/pt/ abstract?short-communication+bn01708042008.

Resumo: A ecologia do golfinho-nariz-de-garrafa (Tursiops truncatus) é pouco documentada no Brasil. O objetivo deste trabalho é fornecer dados sobre a distribuição, tamanho de grupo e comportamento de T. truncatus a partir de avistagens oportunistas realizadas nas adjacências da Ilha de Santa Catarina, sul do Brasil. Em cada observação de grupos da espécie foram registrados: localidade, data e hora, tamanho de grupo e comportamento. Foram observados 71 grupos entre 1989 e 2005 , em todos os meses do ano. A espécie foi observada em toda região costeira da Ilha de Santa Catarina, utilizando uma grande variedade de hábitats. O tamanho de grupo variou entre 1 e 200 indivíduos, com uma média de aproximadamente 8 indivíduos (moda $=2)$. Interações com pelo menos três espécies de aves marinhas também foram observadas, além de outros comportamentos. Os dados obtidos sugerem que a espécie é comum ao longo da costa da Ilha de Santa Catarina, possui uma grande plasticidade no uso de hábitats, além de um conjunto variado de comportamentos.

Palavras-chave: golfinho-nariz-de-garrafa, Tursiops truncatus, comportamento, tamanho de grupo, aves marinhas. 


\section{Introdução}

O golfinho-nariz-de-garrafa (Tursiops truncatus) (Montagu) ocorre tanto em águas costeiras quanto oceânicas, em todas as regiões tropicais e temperadas (Leatherwood \& Reeves 1990). No Brasil a espécie está presente ao longo de toda a costa (Pinedo et al. 1992) e sua aparente ausência na região norte pode estar relacionada ao reduzido esforço amostral. O conhecimento a respeito das populações oceânicas desta espécie em águas brasileiras é praticamente inexistente, constando de observações pontuais (e.g., Baracho et al. 2007).

No litoral do Estado de Santa Catarina a espécie pode ser frequentemente avistada em águas costeiras, penetrando na foz de rios, lagunas e manguezais (Simões-Lopes 1991, Simões-Lopes \& Ximenez 1993). Com base em registros de encalhes ao longo da costa do Estado (Cherem et al. 2004), sua distribuição é aparentemente contínua. No entanto, a maior parte das informações sobre a espécie provém de estudos no litoral sul, direcionados à investigação da interação entre T. truncatus e pescadores artesanais (Simões-Lopes 1991, 1998, Simões-Lopes \& Fabián 1999, Simões-Lopes et al. 1998). Uma população residente de $T$. truncatus pode ser avistada o ano inteiro na entrada do maior complexo lagunar da costa do Estado de Santa Catarina, adjacente à cidade de Laguna (Simões-Lopes \& Fabián 1999). Indivíduos desta população interagem cooperativamente com pescadores artesanais durante, principalmente, a captura da tainha (Mugil sp.), um importante recurso alimentar para a espécie (SimõesLopes et al. 1998). Alguns indivíduos foram observados realizando deslocamentos de até $300 \mathrm{~km}$ de extensão ao longo da costa da região Sul do Brasil (Simões-Lopes \& Fabián 1999), indicando a utilização de extensas áreas por alguns indivíduos da espécie.

Nas imediações da Ilha de Santa Catarina e litoral central do estado, existe a descrição de um evento de interação agressiva entre T. truncatus e o boto-cinza-Sotalia guianensis (van Bénéden) (Wedekin et al. 2004). Excetuando-se este registro pontual, pouco foi relatado sobre a ocorrência e comportamento da espécie nas porções central e norte do Estado de Santa Catarina.

O objetivo deste trabalho é fornecer dados sobre a distribuição, tamanho de grupo e comportamento de T. truncatus a partir de observações oportunistas realizadas nas adjacências da Ilha de Santa Catarina, sul do Brasil.

\section{Material e Métodos}

\section{1. Área de estudo}

A Ilha de Santa Catarina está localizada no litoral central do Estado de Santa Catarina, sul do Brasil, entre as latitudes $26^{\circ}$ e $30^{\circ} \mathrm{S}$, e longitudes $48^{\circ} 30^{\prime}$ e $50^{\circ} \mathrm{W}$ (Figura 1 ). O litoral na qual a Ilha de Santa Catarina está inserida encontra-se dentro da classificação "Costa Granítica do Sudeste" (Ekau \& Knoppers 1999), composto por rochas graníticas do Embasamento Cristalino que formam morros e costões rochosos, intercalados por praias arenosas da Planície Costeira. Assim, o litoral apresenta-se recortado e contendo inúmeras ilhas próximas da costa, sendo a maior delas a Ilha de Santa Catarina, com mais de $400 \mathrm{~km}^{2}$ de área e aproximadamente $170 \mathrm{~km}$ de perímetro de costa. Duas baías de águas protegidas (Baías Sul e Norte) separam a Ilha de Santa Catarina e o continente, e nestas baías é possível encontrar marismas e manguezais.

A costa da Ilha de Santa Catarina é caracterizada por possuir um regime de micromarés (Ekau \& Knoppers 1999) e um ciclo sazonal de temperatura variando entre valores inferiores a $18^{\circ} \mathrm{C}$ no inverno e em torno de $24^{\circ} \mathrm{C}$ no verão (Absy \& Zavialov 1999). A massa de água denominada "Água Costeira" (cf. Matsuura 1986) banha as áreas próximas da Ilha de Santa Catarina, onde as avistagens compiladas neste estudo foram realizadas. Imediatamente ao sul da Ilha de Santa Catarina foi constatada uma área de possível ressurgência costeira em meses quentes do ano (Matsuura 1986).

\section{Coleta e análise dos dados}

Os dados apresentados aqui foram obtidos a partir de esforço de observação oportunista, sem caráter sistemático direcionado à espécie. Na Baía Norte da Ilha de Santa Catarina as observações de T. truncatus foram realizadas a partir de uma embarcação de $5 \mathrm{~m}$ de comprimento durante um estudo sobre a ecologia de $S$. guianensis, entre os anos de 2000 e 2005 (Daura-Jorge et al. 2004, 2005, Wedekin et al. 2007). As outras avistagens foram realizadas por observadores familiarizados com observação de mamíferos marinhos na natureza, entre os anos de 1989 e 2005 , geralmente a partir de pontos em terra. Foram registrados dados como localidade, data e tamanho de grupo. Para as observações de comportamento adotamos o método ad libitum (conforme Lehner 1996).

Cada observação foi plotada em uma carta náutica digitalizada da Ilha de Santa Catarina utilizando o programa ESRI ArcView 3.1.

Um grupo de botos foi definido como sendo qualquer conjunto de indivíduos observados próximos um do outro em aparente associação, geralmente engajados na mesma atividade e se movendo na mesma direção (adaptado de Shane 1990a). Foi considerada uma interação entre botos e aves quando as aves foram observadas voando e/ou mergulhando próximos a um grupo de botos.

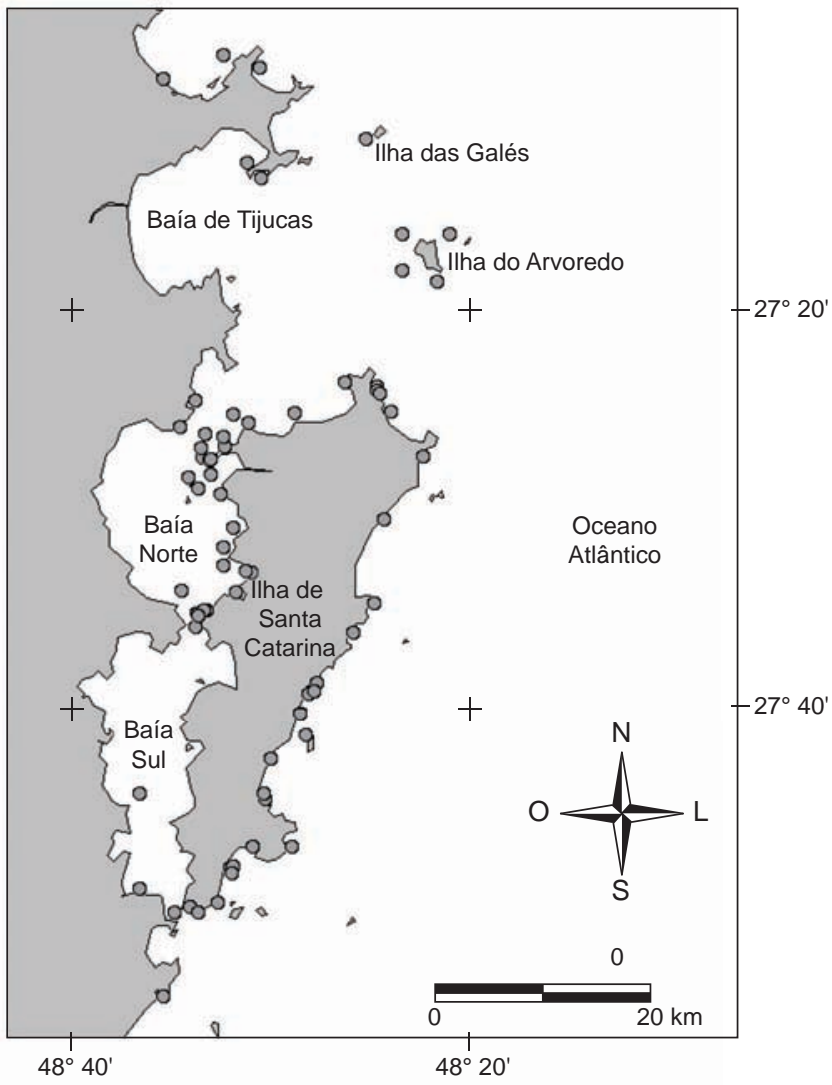

Figura 1. Registros de Tursiops truncatus nas adjacências da Ilha de Santa Catarina entre 1989 e $2005(n=71)$.

Figure 1. Records of Tursiops truncatus in the surroundings of the Island of Santa Catarina between 1989 and $2005(\mathrm{n}=71)$. 


\section{Resultados}

\section{Ocorrência e uso de hábitat}

Foram observados 71 grupos de Tursiops truncatus em toda região costeira da Ilha de Santa Catarina (Figura 1). A espécie foi observada tanto em águas protegidas das Baías Norte e Sul quanto em mar aberto de toda a porção leste da Ilha de Santa Catarina e nas suas porções continentais ao norte e ao sul. A espécie também foi observada nos canais das baías e em áreas mais internas e rasas, como as baías de sedimentação adjacentes aos manguezais do Saco Grande, Ratones e Itacorubi. Alguns registros $(n=5)$ foram realizados próximos de ilhas como Arvoredo e Galés, ao norte da Ilha de Santa Catarina. Outros registros foram realizados próximos a costões rochosos $(n=23)$, manguezais e marismas $(n=3)$, ou perto de zonas de arrebentação de praias arenosas $(n=17)$, na parte leste da ilha. Em pelo menos quatro ocasiões diferentes, a espécie foi observada no meio de cultivos de moluscos, próximo a poitas de embarcações e no cais municipal (trapiche) da Baía Norte. A espécie foi observada em todos os meses do ano (Figura 2).

\section{Comportamento e tamanho de grupo}

Frequentemente $(\mathrm{n}=7)$ foi possível observar T. truncatus em comportamento de alimentação visível da superfície, com saltos, exposição ou batidas de nadadeira caudal, deslocamentos rápidos, perseguições e captura de presas na superfície. Em pelo menos três ocasiões indivíduos de $T$. truncatus foram observados na superfície no momento da captura, com o peixe na boca. A espécie foi observada pescando tanto solitariamente quanto em grupo. Foram observados eventos de captura de presas que variaram de perseguições na zona de arrebentação, próximo de costões rochosos, utilizando a barreira física para arrebanhar cardumes, ou em áreas abertas com até $30 \mathrm{~m}$ de profundidade, sem barreiras ou obstáculos físicos.

O tamanho de grupo variou entre 1 e 200 indivíduos (moda $=2$; média $=8,04 ; \mathrm{DP}=24,35$; Figura 3). O maior grupo, de 200 indivíduos foi observado entre a Ilha do Arvoredo e Deserta. Os maiores grupos avistados em áreas abrigadas foram de 12 indivíduos, na Baía Norte. Grupos menores e indivíduos solitários foram observados em toda a região costeira da Ilha de Santa Catarina. Em apenas quatro registros (7\%) foram avistados grupos contendo pelo menos um filhote.

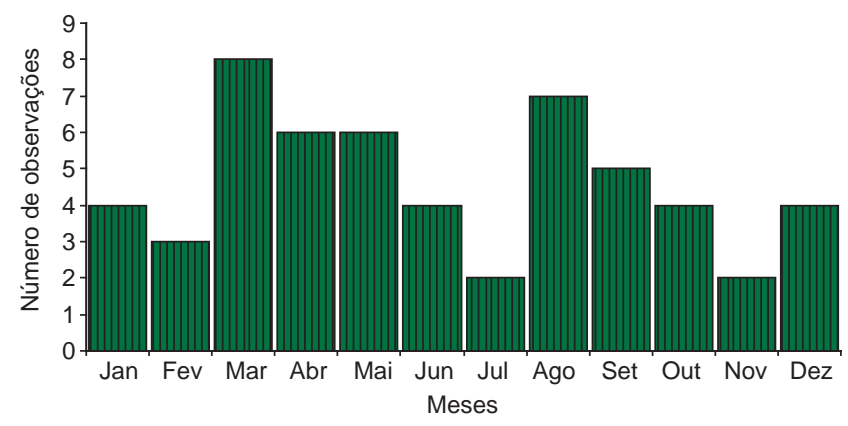

Figura 2. Freqüência absoluta das observações de grupos de Tursiops truncatus nos diferentes meses do ano nas adjacências da Ilha de Santa Catarina entre 1989 e $2005(\mathrm{n}=70)$.

Figure 2. Records of Tursiops truncatus groups in the surroundings of the Island of Santa Catarina by months of the year between 1989 and 2005 $(\mathrm{n}=70)$.

\section{Interações com aves}

Em cinco ocasiões foi possível observar grupos de T. truncatus interagindo com aves marinhas como o atobá-marrom (Sula leucogaster) (Boddaert), fragata (Fregata magnificens) Mathews, e a gaivota (Larus dominicanus) Lichtenstein (Tabela 1). A espécie mais frequentemente avistada interagindo com $T$. truncatus foi S. leucogaster, presente em mais de $80 \%$ dos registros de interação. $\mathrm{Na}$ maioria dos registros de interação $(\mathrm{n}=4 ; 67 \%)$, os grupos de T. truncatus estavam em comportamento de pesca.

\section{Discussão}

Tursiops truncatus foi observado em praticamente todos os ambientes costeiros da Ilha de Santa Catarina, que são equivalentes aos ambientes costeiros de grande parte do litoral catarinense, do extremo norte, na divisa com o Estado do Paraná, até o Cabo de Santa Marta, no sul do Estado (Ekau \& Knoppers 1999). As observações da espécie não estiveram associadas ou concentradas em entradas de rios ou canais das baías conforme se observa na Barra de Laguna (Simões-Lopes \& Fabian 1999) ou na foz do Rio Itajaí-Açú (Lopes et al. 2002). Uma das populações de T. truncatus mais estudadas, da Baía de Sarasota na Flórida, também utiliza uma grande variedade de hábitats, incluindo águas rasas estuarinas e mais afastadas da costa (ver Connor et al. 2000). No Golfo da Califórnia a espécie também foi avistada em diferentes hábitats, incluindo ambientes estuarinos e não estuarinos, apesar de se concentrarem nos primeiros, em locais com fundo de areia e águas mais rasas (Ballance 1992). Para a região do Banco dos Abrolhos, na plataforma continental da costa leste do Brasil, Rossi-Santos et al. (2006) reportaram a ocorrência da espécie como um dos pequenos cetáceos mais abundantes e de distribuição mais ampla na região, freqüentando desde águas costeiras (mas geralmente além dos $12 \mathrm{~km}$ da linha de costa) até pontos mais distantes da costa, de maior profundidade. Sabe-se que T. truncatus é uma espécie generalista em relação ao uso de hábitat, e as avistagens apresentadas aqui também demonstram este aspecto ecológico.

Tursiops truncatus é tido com uma espécie plástica com relação às estratégias de caça e forrageamento (Shane 1990b). O comportamento alimentar de $T$. truncatus pode ser direcionado a peixes solitários ou que andam em cardumes, e as atividades de caça e captura podem ser executadas por botos solitários ou em grupos, em toda a coluna d'água, com variabilidade intra e inter-populacional nas técnicas

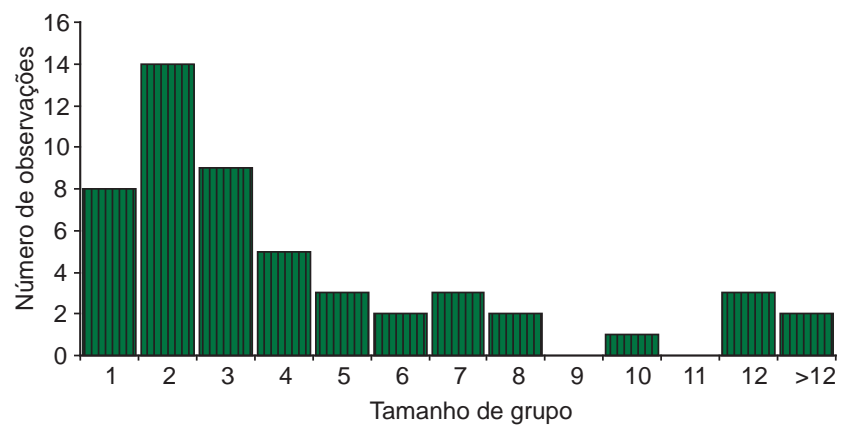

Figura 3. Frequiência absoluta dos tamanhos de grupo registrados nas observações de Tursiops truncatus nas adjacências da Ilha de Santa Catarina entre 1989 e $2005(n=68)$.

Figure 3. Absolute frequency of group sizes recorded for Tursiops truncates in the surroundings of the Island of Santa Catarina between 1989 and 2005 $(\mathrm{n}=68)$. 
Wedekin, L.L. et al.

Tabela 1. Interações entre Tursiops truncatus e aves marinhas registradas nas adjacências da Ilha de Santa Catarina.

Table 1. Interactions between Tursiops truncatus and seabirds observed near the Island of Santa Catarina.

\begin{tabular}{cccc}
\hline Data & Número de botos & Comportamento dos botos & Número de indivíduos e espécie(s) de ave(s) \\
\hline $23 / 05 / 2001$ & 3 adultos & pesca & 3 Fregata magnificens \\
$13 / 01 / 2002$ & 1 adulto & deslocamento & 3 Sula leucogaster \\
$27 / 12 / 2002$ & 12 adultos & pesca & 4 F. magnificens e 1 S. leucogaster \\
$09 / 02 / 2003$ & 4 adultos & pesca & 2 S. leucogaster \\
$27 / 12 / 2003$ & 1 adulto & deslocamento & 4 S. leucogaster e 1 Larus dominicanus \\
$07 / 01 / 2005$ & 10 adultos & pesca & 6 S. leucogaster \\
\hline
\end{tabular}

empregadas pelos animais (Connor et al. 2000). A grande variedade de hábitats que a espécie ocupa em águas adjacentes à costa da Ilha de Santa Catarina, a variação observada no tamanho de grupo, e os eventos de pesca observados, incluindo interações com aves, sugerem que este rico repertório comportamental de alimentação também ocorre no litoral de Santa Catarina.

T. truncatus foi avistado ao longo de todo o ano na costa da Ilha de Santa Catarina. Isto também ocorre na Barra de Laguna, onde se observou que parte da população da espécie é residente (Simões-Lopes \& Fabián 1999). Além das taxas de residência variarem entre diferentes indivíduos, sendo alguns mais residentes que outros, indivíduos foram observados realizando grandes deslocamentos (até $300 \mathrm{~km}$ ) ao longo da costa do Estado de Santa Catarina e do Estado do Rio Grande do Sul (Simões-Lopes \& Fabián 1999). Na costa da Austrália, Corkeron \& Martin (2004) registraram movimentos de até $146 \mathrm{~km}$ para a espécie através de telemetria por satélite. O grau de residência dos grupos observados ao largo da Ilha de Santa Catarina e sua conexão com populações mais austrais ou setentrionais permanecem questões abertas para futuras investigações.

O tamanho de grupo de T. truncatus pode variar bastante entre diferentes localidades, sendo que os encontrados na Ilha de Santa Catarina são próximos das menores médias de tamanho de grupo registradas para a espécie (Connor et al. 2000). Grupos maiores também foram observados em águas abertas e provavelmente trata-se de agregações ocasionais de caráter alimentar, ou ainda, no caso dos grandes grupos avistados próximos da Ilha do Arvoredo (de 200 indivíduos), agregações de uma possível população oceânica da espécie devido a sua distinta coloração (Paulo C. Simões-Lopes, obs. pess.). Isto concorda com a proposta de simpatria de duas subespécies de Tursiops no litoral do Estado de Santa Catarina (Barreto 2000) que têm nichos espaciais diferentes (Barreto \& Beaumord 2002).

A interação de T. truncatus com aves marinhas foi previamente relatada na literatura (e.g. Evans 1982). A ocorrência desta associação durante atividades de alimentação sugere um evento oportunista, porém com considerável importância como estratégia alimentar dentro do repertório comportamental das espécies envolvidas. As mesmas espécies de aves foram observadas interagindo com outra espécie de pequeno cetáceo na área, o boto-cinza, Sotalia guianensis (Rossi-Santos 1997, Piacentini 2003). Esta interação com o boto-cinza está sendo considerada, com base em nossas observações sistemáticas, como uma relação comensal, com benefícios para as aves e neutra para os botos. O mesmo padrão pode ser proposto para T. truncatus na área, apesar dos dados existentes não permitirem uma discussão mais profunda sobre a natureza desta relação.

Os dados apresentados neste trabalho sugerem que a espécie é comum ao longo da região costeira da Ilha de Santa Catarina, possui uma grande plasticidade no uso de hábitats, além de um conjunto variado de comportamentos.

\section{Agradecimentos}

Agradecemos às seguintes pessoas: Alexandre Paro, Daniel dos Santos Lewis, Daniel Rossi Santos, Renato Schultz e Rodrigo Mohedano por gentilmente coletar e ceder dados sobre avistagens da espécie nas praias da Ilha de Santa Catarina. Paulo C. Simões-Lopes agradece a bolsa do CNPq/PQ (processo 304698/2006-7). Dois revisores anônimos fizeram valiosas contribuições ao manuscrito.

\section{Referências Bibliográficas}

ABSY, J.M. \& ZAVIALOV, P.O. 1999. Seasonal cycle of air temperature above the Southwestern Atlantic Ocean. Atlântica 21:11-18.

BALANCE, L.T. 1992. Habitat use patterns and ranges of the bottlenose dolphin in the Gulf of California, Mexico. Mar. Mamm. Sci. 8(3):262-274.

BARACHO, C., CIPOLOTTI, S., MARCOVALDI, E. APOLINÁRIO, M. \& SILVA, M.B. 2007. The occurrence of bottlenose dolphins (Tursiops truncatus) in the biological reserve of Atol das Rocas in north-eastern Brazil. J. Mar. Biol. A. U.K. 2 - Biodiversity Records (Published online).

BARRETO, A.S. 2000. Variação craniana e genética de Tursiops truncatus na costa Atlântica da América do Sul. Tese de Doutorado, Fundação Universidade do Rio Grande, Rio Grande.

BARRETO, A.S. \& BEAUMORD, A.C. 2002. Sobreposição de nicho em subespécies de Tursiops no litoral Atlântico da América do Sul. Notas Téc. FACIMAR 6:121-125.

CHEREM, J.J., SIMÕES-LOPES, P.C., ALTHOFF, S.L. \& GRAIPEL, M.E. 2004. Lista dos mamíferos do estado de Santa Catarina, sul do Brasil. Mastozool. Neotrop. 11(2):151-184.

CONNOR, R.C., WELLS, R.S., MANN, J. \& READ, A.J. 2000. The Bottlenose Dolphin: Social Relationships in a Fission-Fusion Society. In Cetacean Societies: Field Studies of Dolphins and Whales (J. Mann, R.C. Connor, P.L. Tyack \& H. Whitehead, eds.). The University of Chicago Press, Chicago, p. 91-126.

CORKERON, P.J. \& MARTIN, A.R. 2004. Ranging and diving behaviour of two 'offshore' bottlenose dolphins, Tursiops sp., off eastern Australia. J. Mar. Biol. A. U.K. 84(2):465-468.

DAURA-JORGE, F.G., WEDEKIN, L.L. \& SIMÕES-LOPES, P.C. 2004. Variação sazonal da intensidade dos deslocamentos do boto-cinza, Sotalia guianensis (Cetacea: Delphinidae), na Baía Norte da Ilha de Santa Catarina. Biotemas 17(1):203-216.

DAURA-JORGE, F.G., WEDEKIN, L.L., PIACENTINI, V.Q. \& SIMÕESLOPES, P.C. 2005. Seasonal and daily patterns of group size, cohesion and activity of the estuarine dolphin, Sotalia guianensis (P. J. Van Bénéden) (Cetacea, Delphinidae), in southern Brazil. Rev. Bras. Zool. 22(4):1014-1020.

EKAU, W. \& KNOPPERS, B. 1999. An introduction to the pelagic system of the North-East and East Brazilian shelf. Arch. Fish. Mar. Res. 47(2/3):113-132. 
EVANS, P.G.H. 1982. Associations between seabirds and cetaceans: a review Mamm. Rev. 12(1):187-206.

LEATHERWOOD, S. \& REEVES, R.R. (Eds.). 2000. The Bottlenose Dolphin. Academic Press, San Diego.

LEHNER, P.N. 1996. Handbook of ethological methods. 2nd edition. Cambridge University Press, Cambridge.

LOPES, J.P., ALVES, M., BRITTO, M., DEL GOBBO, P., MARCHI, C., RUTHES, A.P., SCHMITZ, A.V., DA SILVA, A.F., BORGES, V., VIERO, S. \& BARRETO, A.S. 2002. Monitoramento de cetáceos no Estuário do Rio Itajaí-Açú (SC, Brasil). In Libro de Resúmenes de la $10^{\mathrm{a}}$ RT y $4^{\circ}$ Congreso SOLAMAC, Valdívia, p. 99.

PIACENTINI, V.Q. 2003. Associação de pesca entre aves marinhas e o botocinza Sotalia guianensis (Cetacea: Delphinidae) na Baía Norte de Santa Catarina, sul do Brasil. Monografia de Bacharelado, Universidade Federal de Santa Catarina, Florianópolis.

MATSUURA, Y. 1986. Contribuição ao estudo da estrutura oceanográfica da região sudeste entre Cabo Frio (RJ) e Cabo de Santa Marta Grande (SC). Ciênc. Cult. 38(8):1439-1450.

PINEDO, M.C., ROSAS, F.C. \& MARMONTEL, M. 1992. Cetáceos e pinnípedes do Brasil: uma revisão dos registros e guia para identificação das espécies. UNEP/FUA, Manaus.

ROSSI-SANTOS, M.R. 1997. Estudo quali-quantitativo do comportamento de alimentação do golfinho ou boto-cinza Sotalia fluviatilis Gervais, 1853 (Cetacea, Delphinidae) na Área de Proteção Ambiental do Anhatomirim e Baía Norte de Santa Catarina. Monografia de Bacharelado, Universidade Federal de Santa Catarina, Florianópolis.

ROSSI-SANTOS, M., WEDEKIN, L.L. \& SOUSA-LIMA, R.S. 2006. Distribution and habitat use of small cetaceans off Abrolhos Bank, eastern Brazil. LAJAM 5(1):23-28.

SHANE, S.H. 1990a. Behavior and ecology of the bottlenose dolphins at Sanibel Island, Florida. In The Bottlenose Dolphin (S. Leatherwood \& R.R. Reeves, eds.). Academic Press, San Diego, p.245-265.
SHANE, S.H. 1990b. Comparison of bottlenose dolphin behavior in Texas and Florida, with a critique of methods for studying dolphin behavior. In The Bottlenose Dolphin (S. Leatherwood \& R.R. Reeves, eds.). Academic Press, San Diego, p.541-558.

SHANE, S.H., REEVES, R.S. \& WÜRSIG, B. 1986. Ecology, behavior and social organization of the bottlenose dolphin: a review. Mar. Mamm. Sci. 2(1):34-63.

SIMÕES-LOPES, P.C. 1991. Interaction of coastal populations of Tursiops truncatus (Cetacea, Delphinidae) with the artisanal fisheries in southern Brazil. Biotemas 4(2):83-94.

SIMÕES-LOPES, P.C. 1998. Intraspecific agonistic behavior of Tursiops truncatus (Cetacea, Delphinidae) during dolphin-human cooperative fishing in southern Brazil. Biotemas 11(2):165-171.

SIMÕES-LOPES, P.C. \& FABIÁN, M.E. 1999. Residence patterns and site fidelity in bottlenose dolphins, Tusiops truncatus (Montagu) (Cetacea, Delphinidae) off Southern Brazil. Rev. Bras. Zool. 16(4):1017-1024.

SIMÕES-LOPES, P.C., FABIÁN, M.E. \& MENEGUETI, J.O. 1998. Dolphin interactions with the mullet artisanal fishing on southern Brazil: a qualitative and quantitative approach. Rev. Bras. Zool. 15(3):709-726.

SIMÕES-LOPES, P.C. \& XIMENEZ, A. 1993. Annotated list of the cetaceans of Santa Catarina coastal waters, southern Brazil. Biotemas 6(1):67-92.

WEDEKIN, L.L., DAURA-JORGE, F.G. \& SIMÕES-LOPES, P.C. 2004. An aggressive interaction between bottlenose dolphins (Tursiops truncatus) and estuarine dolphins (Sotalia guianensis) in southern Brazil. Aq. Mamm. 30(3):391-397.

WEDEKIN, L.L., DAURA-JORGE, F.G., PIACENTINI, V.Q. \& SIMÕESLOPES, P.C. 2007. Seasonal variations in spatial usage by the estuarine dolphin, Sotalia guianensis (van Bénéden, 1864) (Cetacea; Delphinidae) at its southern limit of distribution. Braz. J. Biol. 67(1):1-8.

Recebido em 14/11/07 Versão reformulada recebida em $31 / 10 / 08$ Publicado em 21/11/08 